\title{
Species Richness and Diversity of Resident and Migratory Landbirds in Remnant Forest Patches and Residential Areas in the Florida Keys, USA
}

\author{
Martin B. Main, ${ }^{1}$ Mary C. Christman, ${ }^{2}$ Annisa Karim, ${ }^{3}$ and Mark Hostetler ${ }^{3}$ \\ ${ }^{1}$ Department of Wildlife Ecology and Conservation and Southwest Florida Research and Education Center, University of Florida, \\ 2686 SR 29 North, Immokalee, FL 34142-9515, USA \\ ${ }^{2}$ Department of Statistics, University of Florida, 406 McCarty C, Gainesville, FL 32611-0339, USA \\ ${ }^{3}$ Department of Wildlife Ecology and Conservation, University of Florida, 110 Newins-Zielger Hall, P.O. Box 110430, Gainesville, \\ FL 32611-0430, USA
}

Correspondence should be addressed to Martin B. Main, mmain@ufl.edu

Received 2 January 2011; Revised 16 June 2011; Accepted 23 June 2011

Academic Editor: Madhur Anand

Copyright ( $) 2011$ Martin B. Main et al. This is an open access article distributed under the Creative Commons Attribution License, which permits unrestricted use, distribution, and reproduction in any medium, provided the original work is properly cited.

Conservation of migratory birds necessitates protecting suitable stopover habitat along migratory routes as well as destination habitats, especially near large geographic barriers such as the Gulf of Mexico. The Florida Keys (Keys) are an important stopover and breeding destination for migratory landbirds. We documented 47 migratory and 21 resident landbird species via point counts during March-May 2004 and 2005. As a group, species richness, species diversity, and the effective number of species of migratory landbirds, including several species of conservation concern, was significantly and positively associated with percent cover of tropical hardwood hammock, a threatened upland forest type. The collective resident landbird community in the Keys was negatively associated with native hammock cover, although species diversity of the resident community was positively associated with the proximity of native hammock and several resident species, including species of conservation concern, were commonly or predominantly associated with native hammock. Consequently, conservation of native hammock habitat in the Keys is an important conservation priority for migratory birds and several resident species of conservation concern.

\section{Introduction}

The loss and fragmentation of native plant communities used as breeding and wintering grounds is recognized as a serious threat to the conservation of migratory birds [1-4]. In addition to these destination habitats, the loss of high-quality stopover areas where birds can rest and refuel energy reserves during migration may pose additional challenges to conserving migratory species, especially near large geographic barriers $[5,6]$. Although the role of stopover habitats in migratory bird ecology is not well understood [7], the loss of stopover habitats has been implicated in the decline of some migratory bird populations $[3,4,8]$. Consequently, the availability, location, and quality of both destination and stopover habitats are considered important components of conservation planning for migratory species.
The Florida Keys (Keys) are a chain of low-lying islands that extend from the southeastern tip of Florida and arc in a southwesterly direction to the Dry Tortugas [9]. These islands are located along one of the primary migratory routes for birds that breed in tropical and temperate North America and winter in the Caribbean and South America [10, 11]. The Keys also provide habitat for Caribbean avian species that rarely appear anywhere else in North America, such as the Mangrove Cuckoo (Coccyzus minor), Black-whiskered Vireo (Vireo altiloquus) and the state listed, threatened Whitecrowned Pigeon [11, 12] (Patagioenas leucocephala).

Tropical hardwood hammocks (hammocks) are closed canopy forests characterized by a diverse suite of evergreen and semideciduous woody species primarily of West Indian origin, many or most of which are producers of mast [13, 14]. This forest type has been largely converted to other 


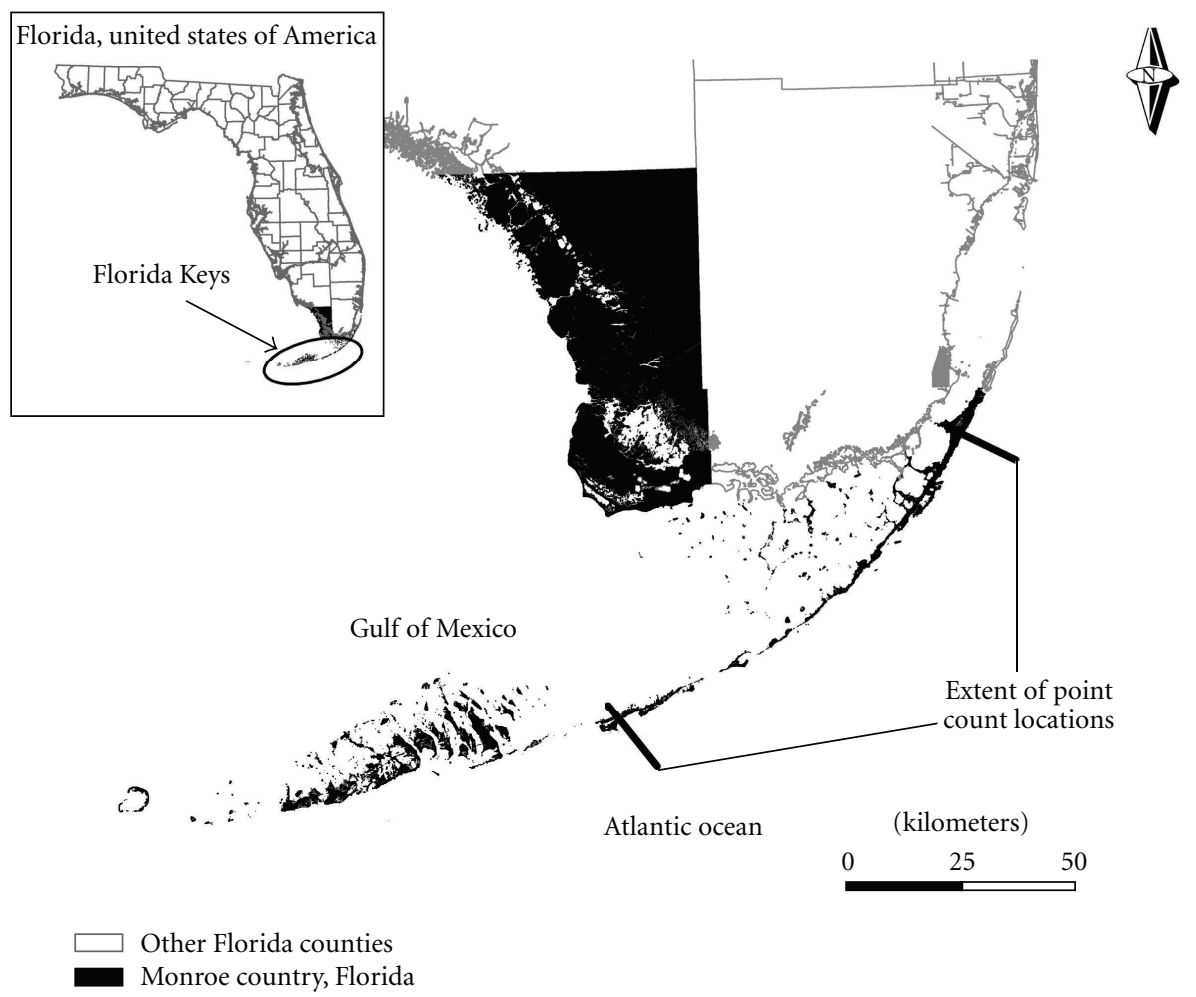

FIGURE 1: Florida Keys archipelago and study area located off the southern tip of Florida, USA.

uses and is ranked as an imperiled habitat type in Florida [11]. Remaining hammock remnants are found primarily in the Keys and those areas in private ownership are under intense development pressure because hammocks occur on the highest elevations where flooding rarely occurs and are prime areas for conversion to residential and urban uses $[15,16]$. Loss of this uniquely situated and rare forest type has potentially serious consequences for many plant and animal species, including migratory and rare Caribbean species of birds that use hammocks for stopover, wintering, and breeding habitat [11].

Although hammocks are believed to provide important habitat for migratory and resident birds [11], little information is available on their use by all but a few species [15], and no information exists to evaluate the comparative value of hammocks and residential areas for migratory and resident bird communities in the Keys. The objective of this study was to quantify the influence of site and landscape variables associated with hammocks and residential areas on species richness and diversity of migratory and resident bird communities in the Keys during the spring migration and breeding period to evaluate the respective roles of hammocks and residential areas as habitat for migratory and resident bird populations.

\section{Methods}

2.1. Study Area. The Keys archipelago (Figure 1) is located within the subtropical region of the Western hemisphere and extends in a southwest direction $354 \mathrm{~km}$ from the southeast tip of peninsular Florida $\left(25^{\circ} \mathrm{N}, 80^{\circ} \mathrm{W}\right)$ to Key West $\left(24^{\circ} \mathrm{N}\right.$, $81^{\circ} \mathrm{W}$ ). The Keys have a mild tropical climate, annual rainfall averages $101 \mathrm{~cm}$, temperatures varying from $10-32^{\circ} \mathrm{C}$, and mean temperature exceeding $18^{\circ} \mathrm{C}$ during all 12 months [21]. The Keys can be distinguished as two geologically separate groups of islands based on substrate (Figure 1). The upper Keys rest on Key Largo Limestone and extend from Soldier Key approximately $150 \mathrm{~km}$ southwest to Big Pine Key, whereas the lower Keys sit on Miami oolitic limestone and extend from Big Pine Key westward approximately $60 \mathrm{~km}$ to Key West [22]. These geological differences influence plant growth, with more and larger hammock communities occurring in the upper Keys [16].

Hammocks in the Keys support high plant diversity and are dominated by evergreen and semi-deciduous woody species, most of which produce mast and are of West Indian origin $[11,13,23]$. Typical canopy species include Gumbolimbo (Bursera simaruba), paradise tree (Simarouba glauca), pigeon-plum (Coccoloba diversifolia), strangler fig (Ficus aurea), wild mastic (Sideroxylon foetidissimum), willowbustic (Sideroxylon salicifolium), and West Indian mahogany (Swietenia mahagoni) among others. Common subcanopy and understory trees and shrubs include Bahama strongbark (Bourreria succulenta), beeftree (Guapira discolor), black ironwood (Krugiodendron ferreum), inkwood (Exothea paniculata), lancewood (Ocotea coriacea), marlberry (Ardisia escallonioides), poisonwood (Metopium toxiferum), satinleaf (Chrysophyllum oliviforme), and red and white stoppers (Eugenia spp.). In addition to hammocks, other native plant communities include mangrove and tidal marsh wetlands 
TABle 1: Description and quantification (mean and standard deviation, median and range) of site and landscape variables at protected and residential sites where bird surveys were conducted (20-m fixed radius point counts) in the Florida Keys during March-May 2004 and 2005.

\begin{tabular}{|c|c|c|c|c|c|c|c|c|c|}
\hline \multirow{2}{*}{ Variable } & \multirow{2}{*}{ Description } & \multicolumn{4}{|c|}{ Protected sites } & \multicolumn{4}{|c|}{ Unprotected sites } \\
\hline & & Mean & $\mathrm{SD}$ & Median & Range & Mean & SD & Median & Range \\
\hline PCT_HAM & $\begin{array}{l}\text { Site variable: percent cover } \\
\text { of native hammock canopy } \\
\text { with intact understory }\end{array}$ & 96 & 8 & 100 & $65-100$ & 18 & 30 & 0 & $0-100$ \\
\hline PCT_CAN & $\begin{array}{l}\text { Site variable: percent cover } \\
\text { of native hammock tree } \\
\text { canopy cover with cleared } \\
\text { understory }\end{array}$ & $<1$ & 1 & 0 & $0-6$ & 18 & 16 & 15 & $0-65$ \\
\hline PCT_LAWN & $\begin{array}{l}\text { Site variable: percent cover } \\
\text { of residential lawn } \\
\text { (turfgrass, ornamental } \\
\text { plantings, pavement, and } \\
\text { structures) }\end{array}$ & 1 & 2 & 0 & $0-10$ & 10 & 11 & 6 & $0-35$ \\
\hline PCT_OTHER & $\begin{array}{l}\text { Site variable: percent cover } \\
\text { of nonhammock vegetation } \\
\text { (e.g., marsh, mangroves, } \\
\text { pinelands) }\end{array}$ & $<1$ & 2 & 0 & $0-8$ & 1 & 5 & 0 & $0-27$ \\
\hline D2HAM & $\begin{array}{l}\text { Landscape variable: } \\
\text { distance }(\mathrm{m}) \text { to closest } \\
\text { hammock (if survey site } \\
\text { was located within a } \\
\text { hammock, D2HAM }=0 \text { ) }\end{array}$ & 0 & 0 & 0 & 0 & 59 & 83 & 26 & $0-417$ \\
\hline SIZEHAM & $\begin{array}{l}\text { Landscape variable: size } \\
\text { (ha) of closest hammock (if } \\
\text { survey site was within a } \\
\text { hammock, the size of that } \\
\text { hammock was recorded) }\end{array}$ & 29 & 30 & 13 & $1-70$ & 7 & 19 & $<1$ & $<1-87$ \\
\hline
\end{tabular}

in low-lying coastal areas, and freshwater marsh and pine (Pinus elliottii) communities in more inland sites [11].

2.2. Bird Surveys. We conducted morning bird surveys between sunrise and 180 minutes after sunrise as $20 \mathrm{~m}$ fixedradius point counts with 5 -min survey intervals [24]. Point count locations were located in conservation areas supporting native hammock communities with intact understory and residential areas with varying degrees of canopy cover during March-May (spring migration and breeding period) 2004 and 2005. Bird surveys were restricted to the upper Keys (Key Largo to Vaca Key, Figure 1) due to logistical constraints and because the upper Keys had more and larger hammock communities than the lower Keys [16].

Point count locations were identified by creating a Geographical Information Systems (ESRI GIS Software ArcMap 9.0) database of all protected hammocks and residential areas [16]. We established point count locations in all protected hammocks in the study area. Residential areas were pooled, numbered, and point count locations were randomly drawn from the dataset of potential sites. Vacant lots dominated by the invasive nonnative shrub Brazilian pepper (Schinus terebinthifolius) were eliminated from the pool of potential survey sites because Brazilian pepper is actively eradicated and represents a temporary condition that is neither a native plant community nor an urbanized habitat type of interest. All point count locations were separated by $>250 \mathrm{~m}$ to maintain independence of observations [24]. Each point count location was marked with flagging tape and location coordinates were recorded with a Global Positioning System (GPS) unit.

We established 86 point count locations, 27 (31\%) of which were in native hammocks and 59 (69\%) of which were in residential areas with varying amounts of native hammock, canopy with open understory, and other cover types. We quantified 4 site variables and 2 landscape variables at each point count location (Table 1 ). We created 20 -m buffers around each point count location (GPS point) in ArcMap and quantified site variables using 2004 digital orthophoto quarter quadrangles (1-m resolution orthorectified aerial images) from the Florida Land Boundary Information System (Florida Department of Environmental Protection, available from http://data.labins.org/2003/, accessed May 2005). We also used ArcMap to measure landscape variables that encompassed areas beyond the 20-m buffer. We groundtruthed all point count locations for accuracy prior to surveys.

We surveyed birds at each point count location 5 times for each field season with the exception of 1 protected hammock where 2 locations were surveyed 4 times during 2004 because permission to access the hammock was delayed. The northern- and southern-most point count locations were separated by approximately $97 \mathrm{~km}$, so we randomly ordered groups of point count locations and alternated the direction 
in which bird surveys were conducted. We conducted point counts at approximately 16 locations per morning.

During surveys, we recorded all birds detected by call, song, or sight within the $20-\mathrm{m}$ point count radius (excluding flyovers) and identified each to species. For each species detected we recorded presence (frequency) and abundance (Table 2). We also calculated detection rates for all species based on frequency (presence/total point counts) and abundance (number birds/total point counts) for point count sites categorized by percent cover of native hammock with understory vegetation (Table 2). Point count site cover categories included $<10 \%$ hammock $(n=42, \bar{x}=1 \%$ cover $)$, $15-52 \%$ hammock $(n=17, \bar{x}=35 \%$ cover $)$ and $65-100 \%$ hammock ( $n=27, \bar{x}=97 \%$ cover $)$.

Each species recorded was classified as either a yearround resident (YR) or as a migratory species (MS), which included migratory species that use the Keys as wintering habitat, as a breeding destination, or as stopover habitat during migration (Table 2). These different categories of migratory birds were pooled because many migratory species could be classified in multiple categories, for example, Gray Catbirds (Dumetella carolinensis) use the Keys as stopover habitat and also winter there [18]. When species included both resident and migratory populations, for example, White-eyed Vireo (Vireo griseus) and Prairie Warbler (Dendroica discolor), the species was designated as YR because it was not possible to differentiate between resident and migrant individuals. For species whose migratory status has been questioned, for example, Black-whiskered Vireo (Vireo altiloquus), Mangrove Cuckoo (Coccyzus minor), and White-crowned Pigeon (Patagioenas leucocephala), we relied upon published authorities to make our determination [17, 18]. Water birds (i.e., seabirds and wetland birds), domestic species (e.g., Peafowl, Pavo cristatus), an unidentified parrot (Amazonia sp.), and 1 rare vagrant (Blue Bunting, Cyanocompsa parellina) were removed from the dataset prior to analysis.

2.3. Statistical Analyses. At each point count location we calculated 3 community measures separately for MS and YR, total species richness, species diversity, and the effective number of species. Species richness was calculated as the total number of species observed at each location. We used the Shannon-Wiener index (Shannon entropy) as an index of species diversity because it is sensitive to changes in the abundance of rare species in a community [25]. We calculated the effective number of species from ShannonWiener index values at each location as $\exp \left(-\sum_{i=1}^{s} p_{i} \ln p_{i}\right)$, according to Jost [26], where $S$ is the total number of species in the community, $i$ is an individual species from that community, and $p_{i}$ is the proportion of $S$ contributed by the $i$ th species. The benefit of calculating the effective number of species is that this measure is described as a true diversity measure that can be calculated from multiple diversity indices (e.g., Shannon-Wiener index, Gini-Simpson index), and the results from these conversions can be compared directly, whereas the original values from different diversity indices cannot be compared [26].
We developed separate heterogeneous slopes models to evaluate the influence of independent landscape and site variables (Table 1) on MS and YR response variables for each community measure (species richness, species diversity, and effective number of species). The independent variables in each model were fit with separate intercepts and slopes for each response variable. In each case we analyzed the influence of independent variables on MS and YR simultaneously in a single model to test whether the MS and YR responded similarly to the habitat variables at each site. In addition to analyzing MS and YR simultaneously, we also analyzed the influence of independent variables on MS and YR in separate models, primarily to evaluate the response of MS and YR separately when the combined models had significant variables that were not group dependent, that is, when the slope of the relationship between the independent variable and the community response was the same for both MS and YR.

In exploratory analyses we found that MS and YR had different error term variances in each case, so we also allowed for unequal variances in the two groups. Further, because the same measures were conducted for MS and YR at all sites, a random block effect for site was included in the model. Interaction terms were used to test whether the slopes of the relationships between each community measure (e.g., species richness) and the independent variables were the same for MS and YR. When the slopes for a particular independent variable are not statistically different this implies that the relationship between the community measure and the independent variable was not statistically different between MS and YR. The subsequent step removed the interaction term and tested whether the slope between diversity and the explanatory variable is nonzero. If there was not a statistically significant relationship, that variable was removed from the model in a stepwise elimination procedure. The final model contained only those explanatory variables found to be statistically significant and also generated the smallest Akaike information criterion (AIC) value of the models tested. Statistical analyses were conducted using the GLIMMIX Procedure in SAS Version 9.2 (Copyright 2008, SAS Institute, Inc.).

\section{Results}

During March-May 2004 and 2005, we conducted 858 point counts at 86 locations and recorded 78 species of birds that numbered 6400 individuals (Table 2). We eliminated 10 species ( 2 domestic, 6 waterbirds, an unidentified parrot, and a rare vagrant) from the data set prior to analysis, which resulted in a final data set of 68 species that totaled 6,260 separate observations (Table 2). Of the 68 species included in the final data set, MS represented 69\% (47 species) and YR 31\% (21 species). Although MS included more than twice the number of species recorded for YR, the number of birds recorded for MS was only 26\% $(n=1,601)$ of total observations recorded for both groups $(\mathrm{YR}=74 \%$, $n=4,659)$.

The response to site and landscape variables (Table 1) differed among MS and YR for some but not all variables 
TABLE 2: Migratory (MS) and year-round resident (YR) species recorded during point counts in the Florida Keys during March-May 2004 and 2005 in residential and conservation areas with varying levels of intact native hammock forest cover. Species are listed from most frequently to least frequently observed during point counts. Total observations are reported for each species as frequency (number of point counts in which species was recorded) and abundance (total number of birds recorded). Percent frequency and percent abundance are provided in parentheses and reflect the relative frequency and abundance of each species within its migratory classification. Detection rates (frequency and abundance) are provided for each species for point count sites categorized by the amount of native hammock cover present: $<10 \%$ (42 sites, $n=420$ point counts), $15-52 \%$ (17 sites, $n=170$ point counts), $65-100 \%$ (27 sites, $n=268$ point counts). Life history details for each species are provided in footnotes. Species with known resident populations were classified as resident species, even if migratory individuals are reported to occur. Classification of migratory and resident status was based on The Birds of North America Online [17] and, Florida Fish and Wildlife Conservation Commission [18]. Nomenclature is consistent with the American Ornithologist's Union Check-list of North American Birds [19].

\begin{tabular}{|c|c|c|c|c|c|c|c|c|}
\hline \multirow[t]{2}{*}{$\begin{array}{l}\text { Species, migratory } \\
\text { classification, and } \\
\text { life-history notes }\end{array}$} & \multicolumn{2}{|c|}{ Total observations } & \multicolumn{3}{|c|}{$\begin{array}{c}\text { Detection rate (frequency) } \\
\text { by } \% \text { hammock cover }\end{array}$} & \multicolumn{3}{|c|}{$\begin{array}{c}\text { Detection rate (abundance) } \\
\text { by } \% \text { hammock cover }\end{array}$} \\
\hline & Frequency $(\%)$ & Abundance (\%) & $<10 \%$ & $15-52 \%$ & $65-100 \%$ & $<10 \%$ & $15-52 \%$ & $65-100 \%$ \\
\hline \multicolumn{9}{|c|}{ Migratory Species (MS) $(n=47)$} \\
\hline $\begin{array}{l}\text { PalmWarbler }{ }^{\mathrm{a}} \\
\text { Dendroica palmarum }\end{array}$ & $157(20)$ & $468(29)$ & 0.186 & 0.182 & 0.179 & 0.624 & 0.453 & 0.481 \\
\hline $\begin{array}{l}\text { Gray Catbird }^{\mathrm{a}} \\
\text { Dumetella carolinensis }\end{array}$ & $149(19)$ & $251(16)$ & 0.171 & 0.153 & 0.190 & 0.312 & 0.277 & 0.272 \\
\hline $\begin{array}{l}\text { Black-whiskered Vireo } \\
\text { Vireo altiloquus }\end{array}$ & $51(7)$ & $63(4)$ & 0.019 & 0.065 & 0.119 & 0.019 & 0.106 & 0.138 \\
\hline $\begin{array}{l}\text { American Redstart } \\
\text { Setophaga ruticilla }\end{array}$ & $48(6)$ & $107(7)$ & 0.043 & 0.065 & 0.071 & 0.098 & 0.118 & 0.172 \\
\hline $\begin{array}{l}\text { Gray Kingbird }{ }^{c} \\
\text { Tyrannus dominicensis }\end{array}$ & $45(6)$ & $87(5)$ & 0.079 & 0.047 & 0.015 & 0.162 & 0.077 & 0.022 \\
\hline $\begin{array}{l}\text { Black-and-white Warbler } \\
\text { Mniotilta varia }\end{array}$ & $44(6)$ & $97(6)$ & 0.019 & 0.047 & 0.105 & 0.038 & 0.124 & 0.224 \\
\hline $\begin{array}{l}\text { Red-eyed Vireo } \\
\text { Vireo olivaceus }\end{array}$ & $38(5)$ & $48(3)$ & 0.012 & 0.018 & 0.112 & 0.014 & 0.018 & 0.146 \\
\hline $\begin{array}{l}\text { White-crowned Pigeon } \\
\text { Patagioenas leucocephala }\end{array}$ & $30(4)$ & $53(3)$ & 0.026 & 0.041 & 0.045 & 0.045 & 0.065 & 0.086 \\
\hline $\begin{array}{l}\text { Ovenbird }^{a} \\
\text { Seiurus aurocapillus }\end{array}$ & $28(4)$ & $31(2)$ & 0.002 & 0.047 & 0.071 & 0.002 & 0.059 & 0.075 \\
\hline $\begin{array}{l}\text { Blue-gray Gnatcatcher } \\
\text { Polioptila caerulea }\end{array}$ & $26(3)$ & $41(3)$ & 0.014 & 0.018 & 0.063 & 0.026 & 0.029 & 0.093 \\
\hline $\begin{array}{l}\text { Blackpoll Warbler }{ }^{\mathrm{b}} \\
\text { Dendroica striata }\end{array}$ & $20(3)$ & $41(3)$ & 0.026 & 0.018 & 0.022 & 0.055 & 0.059 & 0.030 \\
\hline $\begin{array}{l}\text { Common Yellowthroat }{ }^{\mathrm{a}} \\
\text { Geothylpis trichas }\end{array}$ & $15(2)$ & $31(2)$ & 0.007 & 0.047 & 0.015 & 0.014 & 0.106 & 0.026 \\
\hline $\begin{array}{l}\text { Eastern Kingbird Tyrannus } \\
\text { tyrannus }\end{array}$ & $14(2)$ & $17(1)$ & 0.029 & 0.006 & 0.004 & 0.033 & 0.006 & 0.008 \\
\hline $\begin{array}{l}\text { Black-throated Blue } \\
\text { Warbler } \\
\text { Dendroica caerulescens }\end{array}$ & $11(1)$ & $16(1)$ & 0.010 & 0.012 & 0.019 & 0.014 & 0.018 & 0.026 \\
\hline $\begin{array}{l}\text { Cape May Warbler } \\
\text { Dendroica tigrina }\end{array}$ & $11(1)$ & $27(2)$ & 0.010 & 0.012 & 0.019 & 0.017 & 0.012 & 0.067 \\
\hline $\begin{array}{l}\text { Indigo Bunting } \\
\text { Passerina cyanea }\end{array}$ & $11(1)$ & $33(2)$ & 0.019 & 0.018 & 0.000 & 0.057 & 0.053 & 0.000 \\
\hline $\begin{array}{l}\text { Worm-eating Warbler }{ }^{\mathrm{b}} \\
\text { Helmitheros vermivorus }\end{array}$ & $9(1)$ & $10(1)$ & 0.002 & 0.000 & 0.030 & 0.002 & 0.000 & 0.034 \\
\hline $\begin{array}{l}\text { Magnolia Warbler }{ }^{\mathrm{b}} \\
\text { Dendroica magnolia }\end{array}$ & $8(1)$ & $31(2)$ & 0.007 & 0.024 & 0.004 & 0.012 & 0.124 & 0.019 \\
\hline $\begin{array}{l}\text { Blackburnian Warbler }{ }^{\mathrm{b}} \\
\text { Dendroica fusca }\end{array}$ & $6(1)$ & $15(1)$ & 0.005 & 0.018 & 0.004 & 0.019 & 0.035 & 0.004 \\
\hline $\begin{array}{l}\text { Brown-headed Cowbird }{ }^{\text {ad }} \\
\text { Molothrus ater }\end{array}$ & $6(1)$ & $9(1)$ & 0.014 & 0.000 & 0.000 & 0.021 & 0.000 & 0.000 \\
\hline
\end{tabular}


Table 2: Continued.

\begin{tabular}{|c|c|c|c|c|c|c|c|c|}
\hline \multirow[t]{2}{*}{$\begin{array}{l}\text { Species, migratory } \\
\text { classification, and } \\
\text { life-history notes }\end{array}$} & \multicolumn{2}{|c|}{ Total observations } & \multicolumn{3}{|c|}{$\begin{array}{c}\text { Detection rate (frequency) } \\
\text { by } \% \text { hammock cover }\end{array}$} & \multicolumn{3}{|c|}{$\begin{array}{c}\text { Detection rate (abundance) } \\
\text { by } \% \text { hammock cover }\end{array}$} \\
\hline & Frequency $(\%)$ & Abundance (\%) & $<10 \%$ & $15-52 \%$ & $65-100 \%$ & $<10 \%$ & $15-52 \%$ & $65-100 \%$ \\
\hline $\begin{array}{l}\text { Yellow-throated } \\
\text { Warbler }^{\mathrm{a}} \\
\text { Dendroica dominica }\end{array}$ & $6(1)$ & $9(1)$ & 0.005 & 0.012 & 0.008 & 0.010 & 0.012 & 0.011 \\
\hline $\begin{array}{l}\text { Painted Bunting } \\
\text { Passerina ciris }\end{array}$ & $3(<1)$ & $4(<1)$ & 0.005 & 0.000 & 0.004 & 0.007 & 0.000 & 0.004 \\
\hline $\begin{array}{l}\text { Wood Thrush } \\
\text { Hylocichla mustelina }\end{array}$ & $3(<1)$ & $5(<1)$ & 0.002 & 0.000 & 0.008 & 0.005 & 0.000 & 0.011 \\
\hline $\begin{array}{l}\text { Yellow-billed Cuckoo acd } \\
\text { Coccyzus americanus }\end{array}$ & $3(<1)$ & $4(<1)$ & 0.000 & 0.000 & 0.011 & 0.000 & 0.000 & 0.015 \\
\hline $\begin{array}{l}\text { Yellow-rumped Warbler } \\
\text { Dendroica coronata }\end{array}$ & $3(<1)$ & $3(<1)$ & 0.002 & 0.000 & 0.008 & 0.002 & 0.000 & 0.008 \\
\hline $\begin{array}{l}\text { Yellow-throated Vireo } \\
\text { Vireo flavifrons }\end{array}$ & $5(1)$ & $6(<1)$ & 0.007 & 0.000 & 0.008 & 0.010 & 0.000 & 0.008 \\
\hline $\begin{array}{l}\text { American Kestrel }^{\mathrm{a}} \\
\text { Falco sparverius }\end{array}$ & $2(<1)$ & $2(<1)$ & 0.002 & 0.006 & 0.000 & 0.002 & 0.006 & 0.000 \\
\hline $\begin{array}{l}\text { Eastern Phoebe }^{\mathrm{a}} \\
\text { Sayornis phoebe }\end{array}$ & $2(<1)$ & $3(<1)$ & 0.002 & 0.000 & 0.004 & 0.002 & 0.000 & 0.008 \\
\hline $\begin{array}{l}\text { Hooded Warbler }{ }^{\mathrm{b}} \\
\text { Wilsonia citrine }\end{array}$ & $4(1)$ & $6(<1)$ & 0.002 & 0.000 & 0.011 & 0.002 & 0.000 & 0.019 \\
\hline $\begin{array}{l}\text { Merlin }{ }^{\mathrm{a}} \\
\text { Falco sparverius }\end{array}$ & $2(<1)$ & $2(<1)$ & 0.005 & 0.000 & 0.000 & 0.005 & 0.000 & 0.000 \\
\hline $\begin{array}{l}\text { Nashville Warbler } \\
\text { Vermivora ruficapilla }\end{array}$ & $2(<1)$ & $4(<1)$ & 0.002 & 0.000 & 0.004 & 0.007 & 0.000 & 0.004 \\
\hline $\begin{array}{l}\text { Pine Warbler } \\
\text { Dendroica pinus }\end{array}$ & $2(<1)$ & $2(<1)$ & 0.002 & 0.000 & 0.004 & 0.002 & 0.000 & 0.004 \\
\hline $\begin{array}{l}\text { Prothonotary Warbler }{ }^{b e} \\
\text { Protonotaria citrea }\end{array}$ & $2(<1)$ & $2(<1)$ & 0.002 & 0.000 & 0.004 & 0.002 & 0.000 & 0.004 \\
\hline $\begin{array}{l}\text { Ruby-throated } \\
\text { Hummingbird } \\
\text { Archilochus colubris }\end{array}$ & $2(<1)$ & $4(<1)$ & 0.005 & 0.000 & 0.000 & 0.010 & 0.000 & 0.000 \\
\hline $\begin{array}{l}\text { Scarlet Tanager } \\
\text { Piranga olivacea }\end{array}$ & $2(<1)$ & $5(<1)$ & 0.000 & 0.006 & 0.004 & 0.000 & 0.012 & 0.011 \\
\hline $\begin{array}{l}\text { Summer Tanager } \\
\text { Piranga olivacea }\end{array}$ & $2(<1)$ & $4(<1)$ & 0.000 & 0.006 & 0.004 & 0.000 & 0.018 & 0.004 \\
\hline $\begin{array}{l}\text { Swainson's Warbler }{ }^{b} \\
\text { Limnothylpis swainsonii }\end{array}$ & $2(<1)$ & $2(<1)$ & 0.000 & 0.000 & 0.008 & 0.000 & 0.000 & 0.008 \\
\hline $\begin{array}{l}\text { Blue Grosbeak } \\
\text { Guiraca caerulea }\end{array}$ & $1(<1)$ & $2(<1)$ & 0.002 & 0.000 & 0.000 & 0.005 & 0.000 & 0.000 \\
\hline $\begin{array}{l}\text { Cedar Waxwing } \\
\text { Bombycilla cedrorum }\end{array}$ & $1(<1)$ & $45(3)$ & 0.002 & 0.000 & 0.000 & 0.107 & 0.000 & 0.000 \\
\hline $\begin{array}{l}\text { Common Nighthawk }{ }^{\mathrm{c}} \\
\text { Chordeiles minor }\end{array}$ & $1(<1)$ & $1(<1)$ & 0.000 & 0.000 & 0.004 & 0.000 & 0.000 & 0.004 \\
\hline $\begin{array}{l}\text { Northern Parula }^{\mathrm{a}} \\
\text { Parula Americana }\end{array}$ & $1(<1)$ & $4(<1)$ & 0.000 & 0.006 & 0.000 & 0.000 & 0.024 & 0.000 \\
\hline $\begin{array}{l}\text { Northern Rough-winged } \\
\text { Swallow }{ }^{\mathrm{a}} \\
\text { Stelgidopteryx serripennis }\end{array}$ & $1(<1)$ & $1(<1)$ & 0.000 & 0.000 & 0.004 & 0.000 & 0.000 & 0.004 \\
\hline $\begin{array}{l}\text { Rose-breasted Grosbeak } \\
\text { Pheucticus ludovicianus }\end{array}$ & $1(<1)$ & $1(<1)$ & 0.002 & 0.000 & 0.000 & 0.002 & 0.000 & 0.000 \\
\hline $\begin{array}{l}\text { Ruby-crowned Kinglet } \\
\text { Regulus calendula }\end{array}$ & $1(<1)$ & $1(<1)$ & 0.000 & 0.000 & 0.004 & 0.000 & 0.000 & 0.004 \\
\hline
\end{tabular}


Table 2: Continued.

\begin{tabular}{|c|c|c|c|c|c|c|c|c|}
\hline \multirow[t]{2}{*}{$\begin{array}{l}\text { Species, migratory } \\
\text { classification, and } \\
\text { life-history notes }\end{array}$} & \multicolumn{2}{|c|}{ Total observations } & \multicolumn{3}{|c|}{$\begin{array}{c}\text { Detection rate (frequency) } \\
\text { by } \% \text { hammock cover }\end{array}$} & \multicolumn{3}{|c|}{$\begin{array}{c}\text { Detection rate (abundance) } \\
\text { by } \% \text { hammock cover }\end{array}$} \\
\hline & Frequency $(\%)$ & Abundance (\%) & $<10 \%$ & $15-52 \%$ & $65-100 \%$ & $<10 \%$ & $15-52 \%$ & $65-100 \%$ \\
\hline $\begin{array}{l}\text { Tree Swallow }{ }^{\mathrm{a}} \\
\text { Tachycineta bicolor }\end{array}$ & $1(<1)$ & $1(<1)$ & 0.000 & 0.006 & 0.000 & 0.000 & 0.006 & 0.000 \\
\hline $\begin{array}{l}\text { Yellow-bellied } \\
\text { Sapsucker }^{\mathrm{a}} \\
\text { Sphyrapicus varius }\end{array}$ & $1(<1)$ & $1(<1)$ & 0.000 & 0.000 & 0.004 & 0.000 & 0.000 & 0.004 \\
\hline $\begin{array}{l}\text { Yellow-breasted Chat }{ }^{\mathrm{b}} \\
\text { Wilsonia citrine }\end{array}$ & $1(<1)$ & $1(<1)$ & 0.000 & 0.000 & 0.004 & 0.000 & 0.000 & 0.004 \\
\hline $\begin{array}{l}\text { Migratory totals (Freq., } \\
\text { Abun.) and mean } \\
\text { detection rates }\end{array}$ & $784(100)$ & $1601(100)$ & 0.016 & 0.019 & 0.026 & 0.038 & 0.039 & 0.044 \\
\hline \multicolumn{9}{|c|}{ Resident Species (YR) $(n=21)$} \\
\hline $\begin{array}{l}\text { Northern Cardinal } \\
\text { Cardinalis cardinalis }\end{array}$ & $473(20)$ & $962(21)$ & 0.457 & 0.635 & 0.646 & 0.902 & 1.341 & 1.325 \\
\hline $\begin{array}{l}\text { Eurasian Collared Dove } \\
\text { Streptopelia decaocto }\end{array}$ & 455 (19) & $1097(24)$ & 0.757 & 0.618 & 0.119 & 2.000 & 1.229 & 0.179 \\
\hline $\begin{array}{l}\text { Red-bellied Woodpecker } \\
\text { Melanerpes carolinus }\end{array}$ & $381(16)$ & $533(11)$ & 0.574 & 0.477 & 0.220 & 0.857 & 0.629 & 0.246 \\
\hline $\begin{array}{l}\text { Blue Jay } \\
\text { Cyanocitta cristata }\end{array}$ & $177(7)$ & $310(7)$ & 0.298 & 0.229 & 0.049 & 0.514 & 0.400 & 0.097 \\
\hline $\begin{array}{l}\text { Common Grackle } \\
\text { Quiscalus quiscula }\end{array}$ & $159(7)$ & $461(10)$ & 0.245 & 0.212 & 0.075 & 0.738 & 0.729 & 0.101 \\
\hline $\begin{array}{l}\text { Northern Mockingbird } \\
\text { Mimus polyglottos }\end{array}$ & $134(6)$ & $195(4)$ & 0.257 & 0.129 & 0.015 & 0.381 & 0.182 & 0.015 \\
\hline $\begin{array}{l}\text { Mourning Doveg } \\
\text { Zenaida macroura }\end{array}$ & $131(5)$ & $185(4)$ & 0.217 & 0.159 & 0.049 & 0.321 & 0.212 & 0.052 \\
\hline $\begin{array}{l}\text { Great Crested } \\
\text { Flycatcher } \\
\text { Myiarchus crinitus }\end{array}$ & $103(4)$ & $133(3)$ & 0.164 & 0.100 & 0.063 & 0.221 & 0.129 & 0.067 \\
\hline $\begin{array}{l}\text { Red-winged Blackbirdg } \\
\text { Agelaius phoeniceus }\end{array}$ & $101(4)$ & $206(4)$ & 0.157 & 0.082 & 0.078 & 0.383 & 0.135 & 0.082 \\
\hline $\begin{array}{l}\text { White-eyed Vireo } \\
\text { Vireo griseus }\end{array}$ & $88(4)$ & $143(3)$ & 0.005 & 0.077 & 0.272 & 0.005 & 0.129 & 0.444 \\
\hline $\begin{array}{l}\text { European Starling } \\
\text { Sturnus vulgaris }\end{array}$ & $86(4)$ & $250(5)$ & 0.188 & 0.035 & 0.004 & 0.533 & 0.135 & 0.011 \\
\hline $\begin{array}{l}\text { Prairie Warblereg } \\
\text { Dendroica discolor }\end{array}$ & $42(2)$ & $66(1)$ & 0.026 & 0.024 & 0.101 & 0.038 & 0.059 & 0.149 \\
\hline $\begin{array}{l}\text { Red-shouldered Hawk }{ }^{\mathrm{g}} \\
\text { Buteo lineatus }\end{array}$ & $33(1)$ & $38(1)$ & 0.033 & 0.053 & 0.037 & 0.041 & 0.059 & 0.041 \\
\hline $\begin{array}{l}\text { Common } \\
\text { Ground-dove }^{\mathrm{eg}} \\
\text { Columbina passerine }\end{array}$ & $13(1)$ & $16(<1)$ & 0.014 & 0.018 & 0.015 & 0.019 & 0.018 & 0.019 \\
\hline $\begin{array}{l}\text { Northern Flicker } \\
\text { Colaptes auratus }\end{array}$ & $12(1)$ & $15(<1)$ & 0.010 & 0.024 & 0.015 & 0.014 & 0.024 & 0.019 \\
\hline $\begin{array}{l}\text { Mangrove Cuckoo } \\
\text { Coccyzus minor }\end{array}$ & $11(1)$ & $13(<1)$ & 0.000 & 0.000 & 0.041 & 0.000 & 0.000 & 0.049 \\
\hline $\begin{array}{l}\text { White-winged Dove } \mathrm{fg}^{\mathrm{fg}} \\
\text { Zenaida asiatica }\end{array}$ & $9(<1)$ & $14(<1)$ & 0.017 & 0.012 & 0.000 & 0.026 & 0.018 & 0.000 \\
\hline $\begin{array}{l}\text { Carolina Wren } \\
\text { Thryothorus ludovicianus }\end{array}$ & $4(<1)$ & $6(<1)$ & 0.000 & 0.000 & 0.015 & 0.000 & 0.000 & 0.022 \\
\hline $\begin{array}{l}\text { Monk Parakeet }{ }^{\mathrm{f}} \\
\text { Myiopsitta monachus }\end{array}$ & $4(<1)$ & $14(<1)$ & 0.002 & 0.018 & 0.000 & 0.002 & 0.077 & 0.000 \\
\hline
\end{tabular}


TABle 2: Continued.

\begin{tabular}{|c|c|c|c|c|c|c|c|c|}
\hline \multirow[t]{2}{*}{$\begin{array}{l}\text { Species, migratory } \\
\text { classification, and } \\
\text { life-history notes }\end{array}$} & \multicolumn{2}{|c|}{ Total observations } & \multicolumn{3}{|c|}{$\begin{array}{c}\text { Detection rate (frequency) } \\
\text { by } \% \text { hammock cover }\end{array}$} & \multicolumn{3}{|c|}{$\begin{array}{c}\text { Detection rate (abundance) } \\
\text { by } \% \text { hammock cover }\end{array}$} \\
\hline & Frequency $(\%)$ & Abundance (\%) & $<10 \%$ & $15-52 \%$ & $65-100 \%$ & $<10 \%$ & $15-52 \%$ & $65-100 \%$ \\
\hline $\begin{array}{l}\text { Great Horned Owl } \\
\text { Bubo virginianus }\end{array}$ & $1(<1)$ & $1(<1)$ & 0.000 & 0.000 & 0.004 & 0.000 & 0.000 & 0.004 \\
\hline $\begin{array}{l}\text { Yellow Warbler } \\
\text { Dendroica petechial }\end{array}$ & $1(<1)$ & $1(<1)$ & 0.000 & 0.006 & 0.000 & 0.000 & 0.006 & 0.000 \\
\hline $\begin{array}{l}\text { Resident totals (Freq., } \\
\text { Abun.) and mean } \\
\text { detection rates }\end{array}$ & $2418(100)$ & $4659(100)$ & 0.163 & 0.139 & 0.087 & 0.333 & 0.262 & 0.139 \\
\hline $\begin{array}{l}{ }^{a} \text { Neotropical migrant that } \\
\text { b Neotropical migrant that } \\
{ }^{\mathrm{c}} \text { Neotropical migrant that } \\
{ }^{\mathrm{d}} \text { Resident population may } \\
{ }^{\mathrm{e}} \text { Bird of Conservation Con } \\
{ }^{\mathrm{f}} \text { Nonnative species. } \\
\text { gyear-round resident popu } \\
{ }^{\mathrm{h}} \text { Historically considered m } \\
\text { resident population suspec }\end{array}$ & $\begin{array}{l}\text { cludes individuals t } \\
\text { es the Florida Keys } \\
\text { cludes individuals t } \\
\text { established in Flor } \\
\text { rn [20]. } \\
\text { tion exists in Florid } \\
\text { ratory with Florida } \\
\text { d. }\end{array}$ & $\begin{array}{l}\text { hat winter and stopo } \\
\text { exclusively or prima } \\
\text { hat use the Florida K } \\
\text { ida Keys, but majori } \\
\text { a Keys, but migrator } \\
\text { Keys a breeding dest }\end{array}$ & $\begin{array}{l}\text { in the } \mathrm{F} \\
\text { as stope } \\
\text { primari } \\
\text { f indivic }\end{array}$ & $\begin{array}{l}\text { da Keys. } \\
\text { habitat. } \\
\text { s a breedin } \\
\text { ls thought } t \\
\text { so travel to } \\
\text { dence for } n\end{array}$ & $\begin{array}{l}\text { lestination. } \\
\text { e migratory. } \\
\text { eys as a winte } \\
\text { ration is wea }\end{array}$ & estin & & \\
\hline
\end{tabular}

measured and also differed slightly among the 3 community measures (Table 3 ). The two variables that significantly influenced both MS and YR for all 3 community measures (species richness, species diversity, and the effective number of species) were percent cover of native hammock with understory (PCT_HAM) and the size of the nearest hammock (SIZEHAM). Species richness, species diversity, and the effective number of species of MS were all positively associated with increasing PCT_HAM, whereas increasing PCT_HAM was negatively associated with all these measures for YR. The same response to PCT_HAM was observed for all 3 community measures when MS and YR were analyzed in separate models.

SIZEHAM was consistently associated with decreased species richness, diversity, and the effective number of species for both MS and YR (Table 3). That is, as the size of the nearest hammock increased, species richness and diversity decreased. However, unlike PCT_HAM, the relationship of SIZEHAM in the combined analysis was not group dependent, and in each model the group-dependent variable (SIZEHAM* group) was not significant. When the response to SIZEHAM was analyzed in separate models for MS and YR, the relationship remained significant for YR for all 3 community measures (species richness: $F=37.02$, $\mathrm{DF}=1,83, P<0.01$; species diversity: $F=5.53, \mathrm{DF}=1$, $80, P=0.02$; effective species: $F=5.73, \mathrm{DF}=1,80$, $P=0.02$ ), but was not significant for MS for any of the community measures (species richness: $F=1.92, \mathrm{DF}=1$, $83, P=0.17$; species diversity: $F=0.04, \mathrm{DF}=1,80$, $P=0.84$; effective species: $F=0.11, \mathrm{DF}=1,80, P=0.74)$. The combined model results, therefore, indicated that the negative relationship between SIZEHAM and community richness and diversity measures was stronger for YR than MS.

Species diversity and the effective number of species, but not species richness, also decreased for both MS and YR as the distance from the nearest native hammock (D2HAM) increased (Table 3). As with SIZEHAM, the response of MS and YR to D2HAM was similar, and in each model the groupdependent variable (D2HAM* group) was not significant. When analyzed in separate models the relationship was weak for YR (species diversity: $F=2.78, \mathrm{DF}=1,80, P=0.10$; effective species: $F=3.18, \mathrm{DF}=1,80, P=0.08$ ) and was not significant for MS (species diversity: $F=1.76, \mathrm{DF}=1$, $80, P=0.19$; effective species: $F=1.86$, $\mathrm{DF}=1,80, P=$ $0.18)$. These results indicated that the negative relationship between D2HAM and species diversity and effective number of species was not strong, but was stronger for YR than MS.

Percent canopy cover with cleared understory (PCT_CAN) did not significantly influence any community measure for MS or species richness for YR, but was negatively associated with species diversity and effective number of species for YR (Table 3). Percent cover of other habitat types (PCT_OTHERHAB), which on averaged comprised $\leq 1 \%$ of cover types at point count sites (Table 1) did not influence species richness for either MS or YR, but had a negative influence on MS and a positive influence on YR for species diversity, and the opposite results for MS and YR for the effective number of species. The only other variable measured, PCT_LAWN, did not significantly influence species richness, species diversity, or the effective number of species for either MS or YR species.

We documented 8 species (4 MS, 4 YR) listed by the US Fish and Wildlife Service [20] as species of conservation concern and plotted the frequency detection rates for these species at point count sites categorized by percent hammock cover (Figure 2). Although sample sizes were small and the information provided is descriptive, detection rates for the migratory Black-whiskered Vireo were 342\% and $626 \%$ higher at sites with $15-52 \%$ and $65-100 \%$ hammock cover, respectively, than at sites with $<10 \%$ hammock cover. Detection rates for migratory White-crowned Pigeons were also higher (158\% and $173 \%)$ at sites with $15-52 \%$ and 65-100\% hammock cover. Prairie Warblers and Mangrove Cuckoos, both recorded as resident species, had higher 
TABle 3: Heterogeneous slopes model results used to evaluate the influence of site and landscape variables on species richness, species diversity (using Shannon-Wiener index values), and the effective number of species (converted from Shannon-Wiener Index values; [26]) for migratory (MS) and resident (YR) birds, in the Florida Keys during March-May 2004 and 2005. Only the final model solutions and respective Akaike information criterion (AIC) values are presented. Model variables are defined in Table 1.

(a) Species richness final model solutions (AIC $=795.57)$

\begin{tabular}{lcccccc}
\hline Effect & Group ID & Estimate & Std. error & DF & $t$ value & 14.14 \\
\hline Group & MS & 5.04 & 0.36 & 162 & 162 & 39.47 \\
& YR & 9.63 & 0.24 & 162 & -2.35 \\
SIZEHAM & MS \& YR & -0.02 & 0.07 & 162 & 0.01 \\
PCT_HAM* group & MS & 0.03 & 0.01 & 0.01 \\
& YR & -0.03 & 0.01 & 162 & -6.23 & $<0.01$ \\
& & &
\end{tabular}

(b) Species diversity final model solutions (AIC $=203.52$ )

\begin{tabular}{|c|c|c|c|c|c|c|}
\hline Effect & Group ID & Estimate & Std. error & DF & $t$ value & $\operatorname{Pr}>|t|$ \\
\hline \multirow{2}{*}{ Group } & MS & 1.28 & 0.12 & 162 & 10.79 & $<0.01$ \\
\hline & YR & 2.02 & 0.08 & 162 & 26.69 & $<0.01$ \\
\hline D2HAM & MS \& YR & $<-0.01$ & $<0.01$ & 162 & -2.10 & 0.04 \\
\hline SIZEHAM & MS \& YR & $<-0.01$ & $<0.01$ & 162 & -2.14 & 0.04 \\
\hline \multirow{2}{*}{ PCT_CAN*group } & MS & $<0.01$ & $<0.01$ & 162 & 0.79 & 0.43 \\
\hline & YR & -0.01 & $<0.01$ & 162 & -3.35 & $<0.01$ \\
\hline \multirow{2}{*}{ PCT_HAM* group } & MS & $<0.01$ & $<0.01$ & 162 & 2.84 & 0.01 \\
\hline & YR & -0.01 & $<0.01$ & 162 & -7.95 & $<0.01$ \\
\hline \multirow{2}{*}{ CT_OTHERHAB* group } & MS & -0.03 & 0.01 & 162 & -2.47 & 0.02 \\
\hline & YR & 0.01 & 0.01 & 162 & 1.58 & 0.12 \\
\hline
\end{tabular}

(c) Effective number of species final model solutions (AIC $=678.45$ )

\begin{tabular}{|c|c|c|c|c|c|c|}
\hline Effect & Group ID & Estimate & Std. error & DF & $t$ value & $\operatorname{Pr}>|t|$ \\
\hline \multirow{2}{*}{ Group } & MS & 4.06 & 0.49 & 162 & 8.27 & $<0.01$ \\
\hline & YR & 7.25 & 0.35 & 162 & 20.89 & $<0.01$ \\
\hline D2HAM & MS \& YR & $<-0.01$ & $<0.01$ & 162 & -2.24 & 0.03 \\
\hline SIZEHAM & MS \& YR & -0.01 & $<0.01$ & 162 & -2.19 & 0.03 \\
\hline \multirow{2}{*}{ PCT_CAN*group } & MS & 0.01 & 0.02 & 162 & 0.65 & 0.51 \\
\hline & YR & -0.04 & 0.01 & 162 & -3.89 & $<0.01$ \\
\hline \multirow{2}{*}{ PCT_HAM* group } & MS & 0.02 & 0.01 & 162 & 2.63 & 0.01 \\
\hline & YR & -0.04 & $<0.01$ & 162 & -7.97 & $<0.01$ \\
\hline \multirow[t]{2}{*}{ PCT_OTHERHAB* group } & MS & -0.06 & 0.05 & 162 & -1.30 & 0.20 \\
\hline & YR & 0.08 & 0.03 & 162 & 2.50 & 0.01 \\
\hline
\end{tabular}

detection rates at sites where hammock cover was $65-100 \%$, with detection rates being $\geq 388 \%$ for Prairie Warblers, and Mangrove Cuckoos were detected solely at sites with $\geq 65 \%$ hammock cover. The Painted Bunting (Passerina ciris), Prothonotary Warbler (Protonotaria citrea), and Yellow Warbler (Dendroica petechial) each had $\leq 3$ total observations and were observed at sites both with and without substantial hammock cover.

\section{Discussion}

Migratory birds (MS) recorded in the Keys during the spring migration and breeding period were the more species-rich group recorded, with greater than twice as many species as YR. However, in terms of abundance, the number of individuals of YR was nearly 3 times greater than MS. As collective groups, MS and YR species demonstrated different habitat requirements as measured by their response to site and landscape variables. These results illustrate the value of differentiating between migratory and resident species and quantifying community measures such as species richness and diversity measures when evaluating the importance of habitat variables as stopover or destination habitat for migratory birds.

4.1. Migratory Species. The most important finding was the consistent and opposite response of MS and YR, as collective groups of species, to percent cover of native hammock forest with intact understory. Percent cover of native hammock (PCT_HAM) was a significant positive influence on species richness, species diversity, and effective number of species of MS. These results suggest that the majority of migratory 


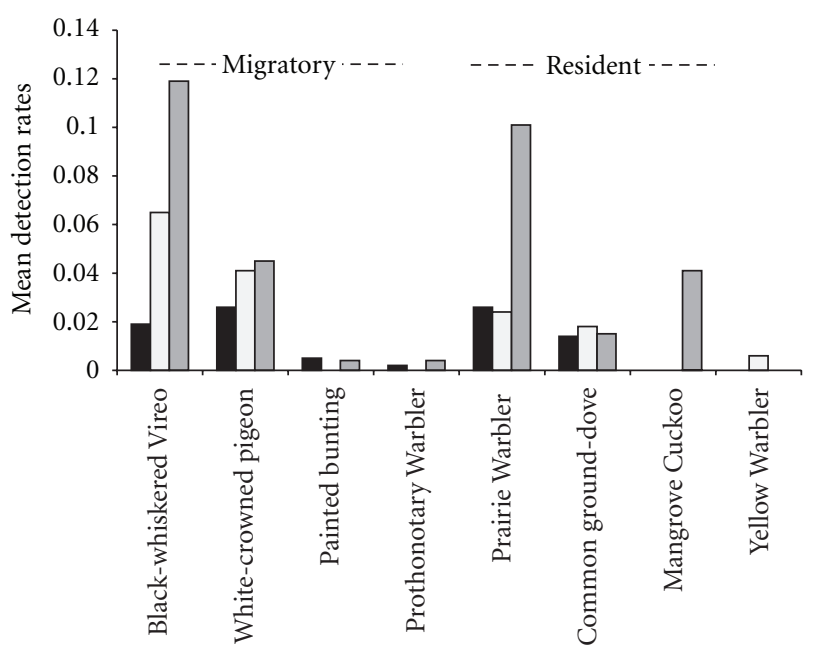

Hammock cover at point count sites (\%):

- $<10 \%$

$\square 15-52 \%$

$\square 65-100 \%$

Figure 2: Mean frequency (presence) detection rates at point count sites categorized by percent hammock cover for migratory and resident species designated as species of conservation concern by the U.S. Fish and Wildlife Service [20].

species recorded in the Keys were dependent upon or preferred this native forest community. This does not mean, however, that all MS rely on native hammock cover. For example, some species such as the Palm Warbler (Dendroica palmarum) and Gray Catbird were commonly recorded at sites with and without native hammock cover, and species that prefer more open habitats, such as the Eastern (Tyrannus tyrannus) and Gray Kingbird ( $T$. dominicensis) were more commonly observed at sites with reduced native hammock cover (Table 2).

Percent canopy cover with cleared understory (PCT_CAN), a site variable associated with residential areas, did not significantly influence species richness, species diversity, or the effective number of species of MS. These results indicate that the availability of tree canopy cover in residential areas cannot provide an adequate substitute for native hammock communities for many MS. However, the absence of a negative influence also suggests tree canopy cover is likely important for many migratory species. A notable example includes the White-crowned Pigeon, which was more often recorded in areas with greater hammock cover but was also observed feeding in the canopy of trees in residential areas with little or no native hammock understory (Figure 2).

The negative relationship between species diversity and the effective number of species of MS and the percent cover of other habitats (PCT_OTHERHAB), such as mangroves, pine, and wetlands needs to be placed in context and should not be interpreted to suggest that these other habitats were unimportant to MS. The objective of this study was to evaluate the role of native hammock cover and residential areas with varying amounts of hammock and tree canopy cover. These other habitat types, while present at many point count sites, averaged $\leq 1 \%$ of the cover at those sites (Table 1 ). Consequently, these habitats were not adequately surveyed to make strong conclusions about their value to MS.

The negative influence of the two landscape variables on species richness and diversity measures for MS includes interpretation that is also somewhat speculative. The findings that species diversity and the effective number of species of MS decreased as the distance to the nearest hammock (D2HAM) increased seem straightforward and suggest that MS were attracted to hammocks and numbers of MS were greater in surrounding areas near hammocks. This interpretation is consistent with the influence of site variables, that is, that MS were positively associated with hammock cover. However, the negative relationship between the size of the nearest hammock (SIZEHAM) and species richness and diversity measures for MS is less clear. Because SIZEHAM included the hammock being sampled when point count sites were located within a hammock (Table 1), we suspect these results were an artifact of greater dispersion and reduced detection of MS in larger hammocks. Hammocks are densely vegetated and birds in larger hammocks are likely more dispersed and detection rates lower. What is clear from our analyses is that native hammocks are important to MS, and previous work on conservation strategies, edge effects, and species-area relationships [27, 28] all support the principle that larger hammocks are ultimately more valuable as habitat for MS and for conserving biodiversity than smaller hammocks.

4.2. Year-Round Residents. Species richness, species diversity, and effective number of species measures for YR responded in opposite ways to the amount of native hammock cover (PCT_HAM) observed for MS. Whereas PCT_HAM was the most important variable influencing presence of MS, this variable had a negative influence on the collective population of YR. This is because the majority of YR documented included species that are commonly associated with residential areas (Table 2). These included 2 nonnative species, the Eurasian Collared Dove (Streptopelia decaocto) and European Starling (Sturnus vulgaris), which are commonly associated with urban landscapes [29] and represented the most abundant and the sixth most abundant YR observed, respectively.

These results do not imply that there were no YR species that use or may even require native hammock cover. On the contrary, several YR species, such as the White-eyed Vireo and Carolina Wren (Thryothorus ludovicianus) were found almost exclusively in hammocks. Resident species that preferred native hammock cover included the Prairie Warbler and Mangrove Cuckoo, both of which are species of conservation concern, and both of which were observed primarily or exclusively in hammocks (Figure 2). In fact, the Mangrove Cuckoo is reported to be dependent upon native hammock and mangrove communities for foraging and breeding habitat [18]. Consequently, native hammock habitat is important to some species of YR. Likewise, other 
habitat types (PCT_OTHERHAB) contributed positively to YR species diversity measures and some species (e.g., Redwinged Blackbird, Agelaius phoeniceus), were most commonly observed in habitat types other than hammock or strictly residential landscapes.

As with MS, a negative relationship existed between SIZEHAM and D2HAM for species richness and diversity measures for YR. The negative relationship with SIZEHAM is consistent both with the observation that most YR species avoided hammocks and that detection rates were likely lower in larger hammocks due to dispersion and detection issues. Although detection rates were not quantified between YR and MS or between hammocks and residential areas, we do not believe this influences our conclusions. The negative relationship of both MS and YR (when analyzed simultaneously) with SIZEHAM does not alter interpretations of the positive response of MS and the negative response of YR to PCT_HAM, because YR species were much more abundant than MS and if YR were using hammocks to a similar or greater extent than MS, our data would have reflected this.

Although species richness and diversity measures for YR were negatively associated with PCT_HAM, we contend that the relationship between species diversity measures for YR and the distance to the nearest hammock (D2HAM) indicates most YR species benefit from the proximity of hammocks. Increased habitat diversity and foraging opportunities associated with native hammocks likely increased species diversity of YR in areas near hammocks, even if those species were more often observed in the residential landscape. This is because generalist species such as the Northern Mockingbird (Mimus polyglottos) and Blue Jays (Cyanocitta cristata) and species that prefer more open habitat (e.g., Great Crested Flycatcher, Myiarchus crinitus) likely benefited from the structural diversity, foraging, and nesting opportunities provided by edge habitat associated with hammocks, and several YR species, such as the Northern Cardinal (Cardinalis cardinalis) and Red-bellied Woodpecker (Melanerpes carolinus) were regularly documented in both areas with and without native hammock cover (Table 2).

4.3. Conservation Implications. Conservation planning often prioritizes the protection of large, contiguous natural and seminatural areas to maximize benefits to biodiversity and sustain ecological processes and function [28]. These principles, however, may in some situations need to be scaled down to prioritize protection of remaining remnants of natural communities that provide important ecological services $[7,16]$. The protection of important stopover and destination habitats used by migratory birds may fall into this type of conservation planning because there is evidence that bottlenecks in the amount and/or quality of stopover habitat may have negative impacts on migrant populations and the importance of conserving wintering and breeding habitat is well documented [3,4]. Although much remains unknown regarding stopover habitat selection, it is clear that the availability and quality of stopover habitat may be critical for successful migration and reproduction, especially for migratory birds crossing major geographic barriers such as the Gulf of Mexico $[6,30]$ or seeking temporary stopover habitat in modified landscapes [29, 31].

Our results documented the importance of native hammock forest remnants to migratory birds in the Keys, including several species of conservation concern and provide empirical support for conservation efforts to protect remaining hammock habitat [16], such as the Florida Keys Ecosystem project [32]. Furthermore, although the majority of YR were garden-variety species commonly associated with residential areas, the proximity of native hammock habitat increased species diversity measures for YR, and several YR species were found primarily in native hammocks, including the Mangrove Cuckoo and resident populations of Prairie Warblers, both of which are species of conservation concern [20]. Consequently, conservation of native hammock remnants in the Keys is an appropriate conservation priority for conserving both resident and migratory bird populations.

\section{Acknowledgments}

The authors thank the Islamorada Village of Islands for providing funding for this project. They also thank the staff and volunteers of Windley Key Fossil Reef Geological State Park and the staff at the Keys Marine Laboratory for their helpfulness during the fieldwork portion of their research.

\section{References}

[1] S. R. Morris, D. W. Holmes, and M. E. Richmond, "A ten-year study of the stopover patterns of migratory passerines during fall migration on Appledore Island, Maine," Condor, vol. 98, no. 2, pp. 395-409, 1996.

[2] F. R. Moore, "Preface," in Stopover Ecology of NearcticNeotropicallandbird Migrants: Habitat Relations and Conservation Implications, F. R. Moore, Ed., Studies in Avian Biology 20, pp. 1-3, Cooper Ornithological Society, 2000.

[3] J. Faaborg, R. T. Holmes, A. D. Anders et al., "Conserving migratory land birds in the New World: do we know enough?" Ecological Applications, vol. 20, no. 2, pp. 398-418, 2010.

[4] J. Faaborq, R. T. Holmes, A. D. Anders et al., "Recent advances in understanding migration systems of New World land birds," Ecological Monographs, vol. 80, no. 1, pp. 3-48, 2010.

[5] F. R. Moore, P. Keplinger, and T. R. Simons, "Stopover on a Gulf Coast barrier island by spring trans-gulf migrants," Wilson Bulletin, vol. 102, pp. 487-500, 1990.

[6] D. W. Mehlman, S. E. Mabey, D. N. Ewert et al., "Conserving stopover sites for forest-dwelling migratory landbirds," Auk, vol. 122, no. 4, pp. 1281-1290, 2005.

[7] D. R. Petit, "Habitat use by landbirds along NearcticNeotropical migration routes: implications for conservation of stopover habitats," in Stopover Ecology of NearcticNeotropicallandbird Migrants: Habitat Relations and Conservation Implications, F. R. Moore, Ed., Studies in Avian Biology 20, pp. 15-33, Cooper Ornithological Society, 2000.

[8] W. C. Barrow Jr., C. Chen, R. B. Hamilton, K. Ouchley, and T. J. Spengler, "Disruption and restoration of en route habitat, a case study: the Chenier Plain," in Stopover Ecology of Nearctic-Neotropicallandbird Migrants: Habitat Relations and Conservation Implications, F. R. Moore, Ed., Studies in Avian Biology 20, pp. 71-87, Cooper Ornithological Society, 2000. 
[9] Florida Department of Community Affairs, "Final report of the Florida keys carrying capacity study implementationrule28-20 work group," Florida State University, Tallahassee, Fla, USA, 2003.

[10] F. C. Lincoln, S. R. Peterson, and J. L. Zimmerman, "Migration of birds," U.S. Department of the Interior, U.S. Fish and Wildlife Service, Washington, DC, USA, 1998, Circular 16. (Version 02APR2002), 1998, http://www.npwrc.usgs.gov/ resource/othrdata/migratio/migratio.htm.

[11] United States Fish and Wildlife Service, "South Florida multispecies recovery plan," United States Fish and Wildlife Service, South Florida Ecological Services Office, Vero Beach, Fla, USA, 2007, http://www.fws.gov/verobeach/index.cfm?Method =programs\&NavProgramCategoryID=3\&programID=107\& ProgramCategoryID $=3$.

[12] R. C. Banks, C. Cicero, J. L. Dunn et al., "Forty-fourth supplement to the American Ornithologists' Union check-list of North American birds," Auk, vol. 120, no. 3, pp. 923-931, 2003.

[13] J. R. Snyder, A. Hernand, and W. B. Robertson Jr., "South Florida rocklands," in Ecosystems of Florida, L. Myers and J. J. Ewel, Eds., pp. 230-277, University of Central Florida Press, Florida, Fla, USA, 1990.

[14] A. Karim and M. B. Main, "Tropical hardwood hammocks in Florida," University of Florida Cooperative Extension Service Document WEC 206.4 pp. University of Florida, UF/IFAS Extension Digital Information Source (EDIS) Database, 2004, http://edis.ifas.ufl.edu/UW206.

[15] G. T. Bancroft, A. M. Strong, and M. Carrington, "Deforestation and its effects on forest-nesting birds in the Florida keys," Conservation Biology, vol. 9, no. 4, pp. 835-844, 1995.

[16] A. Karim and M. B. Main, "Habitat fragmentation and conservation strategies for a rare forest habitat in the Florida Keys archipelago," Urban Ecosystems, vol. 12, no. 3, pp. 359370, 2009.

[17] A. Poole, Ed., The Birds of North America Online, Cornell Lab of Ornithology, Ithaca, NY, USA, 2010, http://bna.birds .cornell.edu/bna/.

[18] Florida Fish and Wildlife Conservation Commission, "Florida's breeding bird atlas: a collaborative study of Florida's birdlife," 2003, http://www.myfwc.com/bba/.

[19] The American Ornithologist's Union Check-List of North American Birds, American Ornithologist's Union, 7th edition, 2008, http://www.aou.org/checklist/north/print.php/.

[20] United States Fish and Wildlife Service, "Birds of conservation concern 2008," United States Department of Interior, Fish and Wildlife Service, Division of Migratory Bird Management, Arlington, Va, USA, 2008, http://www.fws .gov/migratorybirds/.

[21] Florida Climate Center, "The center for ocean-atmospheric prediction studies," Florida State University, Tallahassee, Fla, USA, 2005, http://coaps.fsu.edu/climate_center.

[22] R. B. Brown, E. L. Stone, and V. W. Carlisle, "Soils," in Ecosystems of Florida, R. L. Myers and J. J. Ewel, Eds., pp. 3569, University of Central Florida Press, Orlando, Fla, USA, 1990.

[23] T. E. Lodge, The Everglades Handbook-Understanding the Ecosystem, CRC Press, Boca Raton, Fla, USA, 2nd edition, 2005.

[24] R. L. Hutto, S. M. Pletschet, and P. Hendricks, "A fixed-radius point count method for nonbreeding and breeding season use," Auk, vol. 103, no. 3, pp. 593-602, 1986.
[25] A. R. Solow, "A simple test for change in community structure," Journal of Animal Ecology, vol. 62, no. 1, pp. 191193, 1993.

[26] L. Jost, "Entropy and diversity," Oikos, vol. 113, pp. 363-375, 2006.

[27] G. K. Meffe, C. R. Carroll et al., Principles of Conservation Biology, Sinauer Associates, Sunderland, Mass, USA, 1994.

[28] R. F. Noss, M. A. O’Connell, and D. D. Murphy, The Science of Conservation Planning: Habitat Conservation under the Endangered Species Act, Island Press, Washington, DC, USA, 1997.

[29] M. E. Hostetler and C. S. Holling, "Detecting the scales at which birds respond to landscape structure in urban landscapes," Urban Ecosystems, vol. 4, pp. 25-54, 2000.

[30] S. A. Gauthreeaux Jr., "Neotropical migrants and the Gulf of Mexico: the view from aloft," in Gatherings of Angels: Migrating Birds and Their Ecology, K. P. Able, Ed., pp. 27-49, Cornell University Press, Ithaca, NY, USA, 1999.

[31] M. Hostetler, S. Duncan, and J. Paul, 'The effects of an apartment complex on migrating and wintering birds," Southeastern Naturalist, vol. 4, no. 3, pp. 421-434, 2005.

[32] Florida Department of Environmental Protection, "Florida keys ecosystem," in Florida Forever Five Year Plan, pp. 180-194, Florida Department of Environmental Protection Division of State Lands Office of Environmental Services, 2010, http:// www.dep.state.fl.us/lands/FFAnnual/EntireRpt_0910.pdf. 

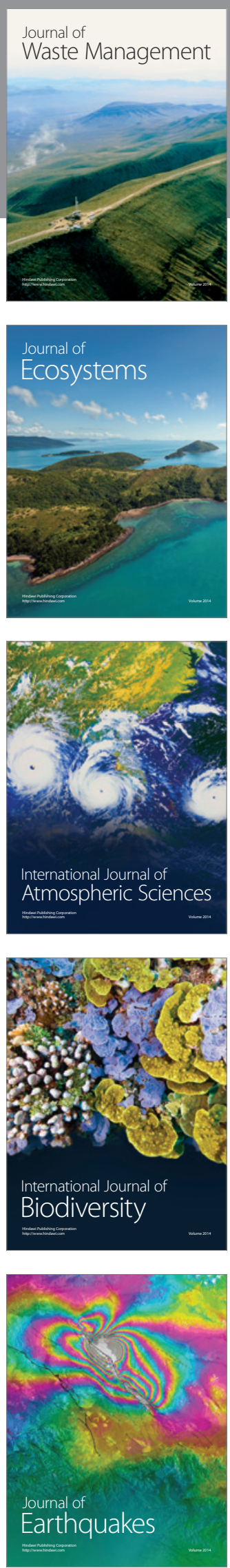
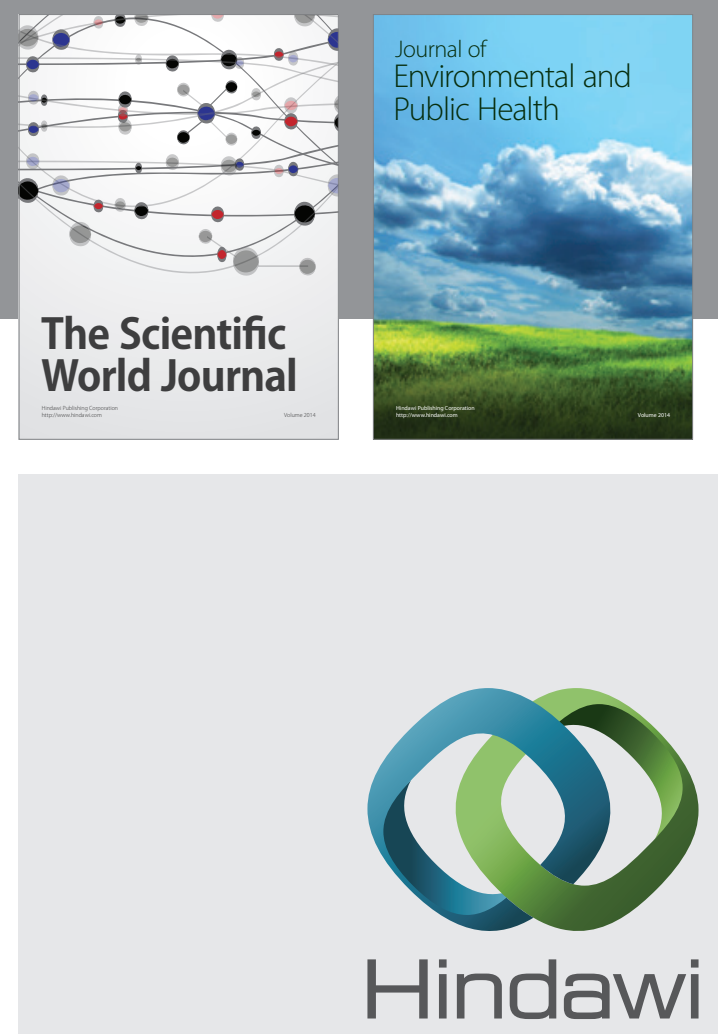

Submit your manuscripts at

http://www.hindawi.com
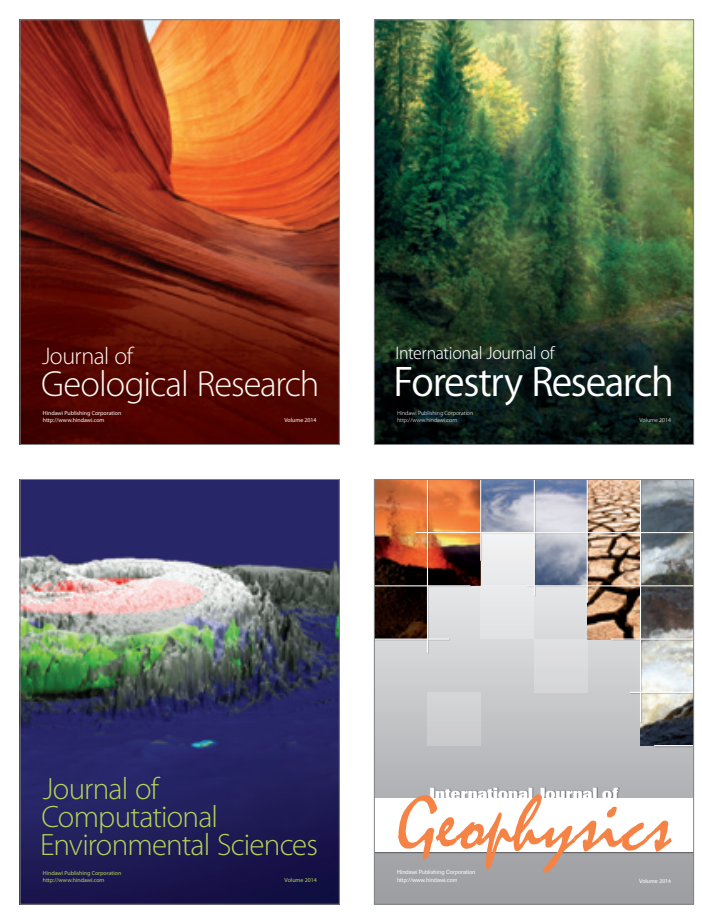
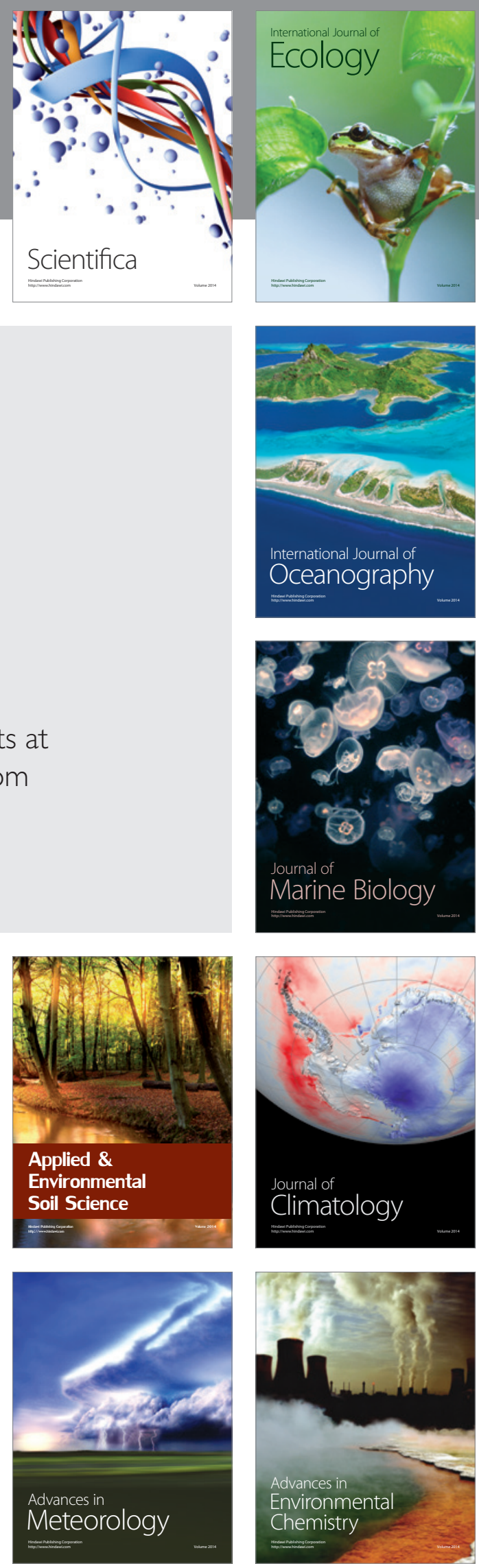